\title{
Nanofluidic Tuning of Photonic Crystal Circuitry
}

\author{
David Erickson, Troy Rockwood, Teresa Emery, Axel Scherer and Demetri Psaltis \\ Center for Optofluidic Integration, California Institute of Technology, Pasadena, California, 91125 \\ de54@cornell.edu, troyrock@caltech.edu, the1@caltech.edu, etcher@caltecu.edu, psaltis@optics.caltech.edu
}

Utilizing soft-lithography based nanofluidics and silicon nanophotonics we demonstrate ultracompact tunable spectral filtering. Liquid based addressing and high refractive index modulation of a single row of holes within a planar photonic crystal is demonstrated.

(C) 2006 Optical Society of America

OCIS codes: 230.7370 Waveguides, 250.5300 Photonic integrated circuits, 999.9999 Nanofluidics

Nanofluidics provides a solution that enables localized control and high refractive index modulation. We demonstrate the integration of multi-layer soft-lithography [1] nanofluidics with silicon nanophotonics and use it to address and tune features within a planar photonic crystals [2]. We introduce pre-engineered defects into an otherwise regular lattice to create a spectrally selective waveguide. This is the first step in the developmental goal of two dimensional reconfigurable photonic circuits.

Figure 1 outlines our approach which follows a three level architecture: the nanophotonic level, the nanofluidic delivery level (which delivers liquids directly into the photonic structure) and the microfluidics control engine (which performs all fluidic manipulations). The photonic level consists of an array of 30 identical photonic crystal structures defined through electron beam lithography and dry etching in a silicon-on-insulator, SOI, substrate with a triangular lattice of holes $(\mathrm{a}=434 \mathrm{~nm}, \mathrm{r}=140 \mathrm{~nm}, \mathrm{~h}=207 \mathrm{~nm})$. We defined the pattern in a negative flowable oxide resist which is left on after processing to enhance bonding with the fluidic layer [3]. Ridge waveguides extend from the crystal to the edge of the chip for optical coupling. In the experiments presented here we increase the radius of the holes within the central row of the photonic crystal (which is to be targeted fluidically) to $203 \mathrm{~nm}$ in order to introduce a reduced index guided mode into the photonic crystal band gap.

(a)

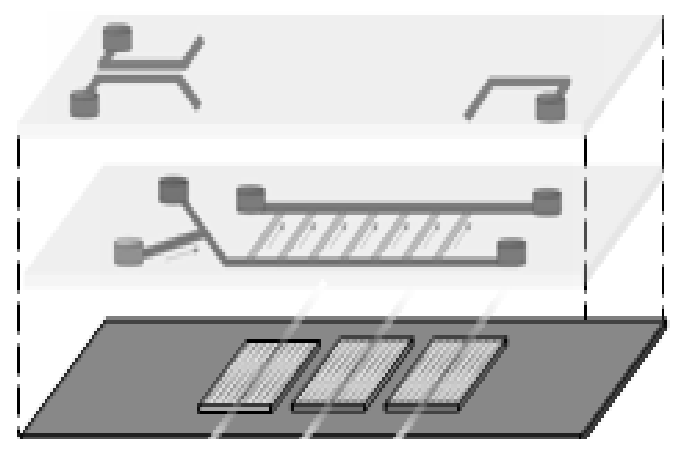

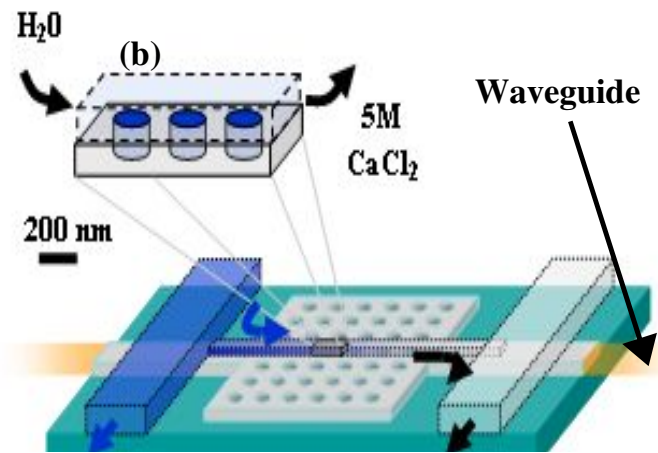

Fig. 1: Nanofluidically tunable photonic structures. (a) Exploded view of optofluidic assembly showing photonic layer on bottom, fluidic layer in the middle and control layer on top. (b) Overview of device operation. The microfluidic control engine mixes and dispenses liquid plugs to the nanofluidic array. The nanofluidic structure serves to deliver liquids directly into a targeted row of holes in the photonic crystal enabling localized [at sub-wavelength scales], high $\mathrm{n} / \mathrm{n}$ refractive index tuning. The optical response is changed by replacing the $5 \mathrm{M} \mathrm{CaCl}_{2}$ solution within $\mathrm{H}_{2} \mathrm{O}$ and vice versa.

Liquids selected for dynamic modulation of the refractive index within the photonic structure must exhibit relatively low viscosity and high $\mathrm{n}^{\text {liquids }} / \mathrm{n}^{\text {substrate }}$. We selected DI (deionized) water $(\mathrm{n}=1.33)$ and aqueous $5 \mathrm{M} \mathrm{CaCl}^{2}$, $(\mathrm{n}=1.44)$ [4]. Figure 2a shows the normalized quasi-TE mode transmission through the photonic crystal for both using a tunable infrared laser source the output which was coupled into the ridge waveguide using a tapered fiber lens. The output coupled back into a second tapered lens fiber and recorded on an optical power meter. The data 


\section{CWK5.pdf}

presented has been smoothed to remove higher frequency Fabry-Perot resonances. The results show a shift in the peak transmission of the guided mode from a/ $=0.291$ to a/ $=0.289$ ( $=1491 \mathrm{~nm}$ and $1502 \mathrm{~nm}$ respectively.) The higher index solution serves to decrease the effective optical radius of the holes and shifts the guided mode towards the dielectric band of the regular crystal. The high $\mathrm{n} / \mathrm{n}$ afforded by nanofluidic modulation demonstrated here enables consistently high contrast over a relatively wide range of wavelengths.
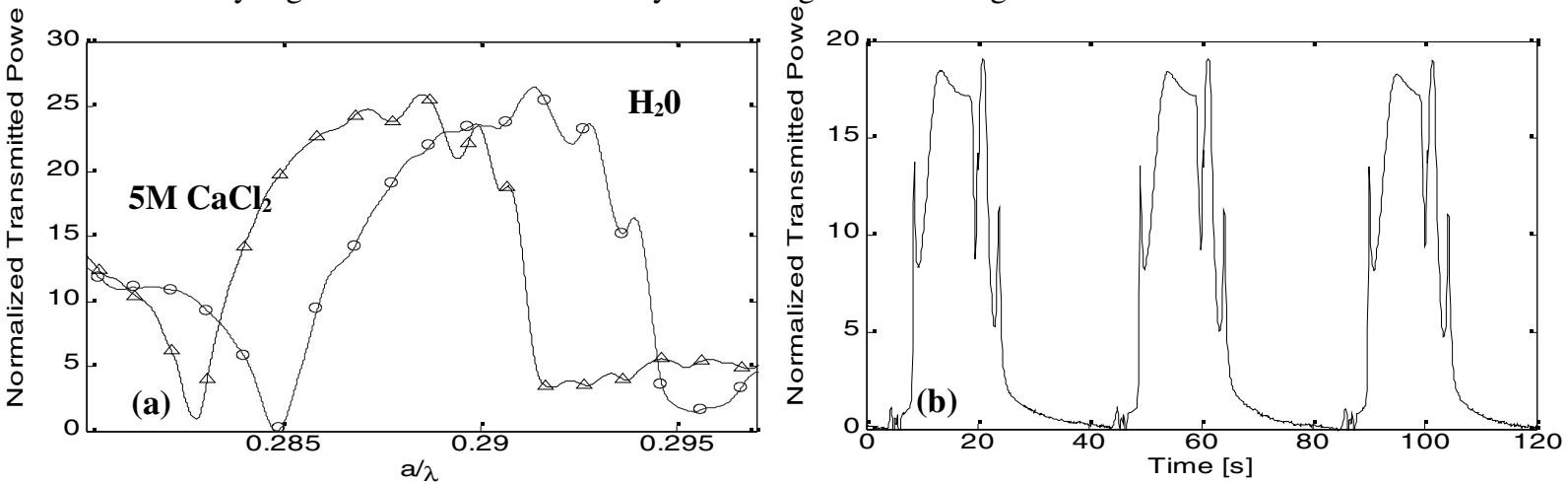

Fig. 2: TE-like transmission through photonic crystal with aligned nanochannel. (a) Shift in transmitted power spectrum when aligned nanochannel filled with $\mathrm{H}_{2} \mathrm{O}(\mathrm{n}=1.33)$ and $5 \mathrm{M} \mathrm{CaCl}_{2}(\mathrm{n}=1.44)$ (b) Dynamic switching at a/ $=0.291$.

Dynamic modulation of the transmitted power is demonstrated in Fig. $2 \mathrm{~b}$ at $\mathrm{a} /=0.291$ by fluidically switching between the DI water and $\mathrm{CaCl}^{2}$ solutions. The switching time is roughly on the second timescale. Simple laminar flow analysis shows that minimum pressure driven transport switching time, scales roughly with $\mathrm{P}$ $\mathrm{L}^{2} / \mathrm{D}^{\mathrm{h}^{2}}$ (where $\mathrm{P}, \mathrm{L}$ and $\mathrm{D}^{\mathrm{h}}$ are the applied pressure, channel length and hydraulic diameter respectively). While $\mathrm{L}$ could be reduced, potentially bringing the switching time down into the millisecond range, $\mathrm{D}^{\mathrm{h}}$ represents the a fundamental limitation on the speed as the geometry is fixed by the lattice constant of the photonic crystal. Electrokinetic transport exhibits a more favorable scaling ratio independent of $\mathrm{D}^{\mathrm{h}}, \mathrm{t}^{\mathrm{s}} \quad \mathrm{V} \mathrm{L}^{2}$ which could result in lower switching times, however, the low electroosmotic mobility of the $\mathrm{CaCl}^{2}$ solution made such an approach less attractive. The diffusive transport timescale into the nanowells $\left(t^{d} d^{2} / D\right.$ where $d$ is well depth and $D$ is the diffusion coefficient) is on the order of $10^{-6} \mathrm{~s}$. The reproducible peaks in output power shown in Fig. $2 \mathrm{~b}$ are a result of irregular bumps in the transmission spectrum of the photonic crystal as they pass through the switching wavelength.

The integration of nanofluidics with nanophotonics presents a new approach for dynamic manipulation of optical properties at sub-wavelength length scales. Extensions of this technique could be used create fully reconfigurable photonic devices through arbitrary redefinition of fluidically defined defects (i.e. passive structures could be activated or defined fluidically when required and then removed later in favor of alternative functionality). Other potential functionalities include delivery of optical gain media, non-linear liquids, or colloidal particles into arbitrary regions of these structures. Such integration could also enable a new class of resonant cavity sensors incorporating targeted delivery of single or few molecules.

\section{References}

1. M. Unger, H. Chou, T. Thorsen, A. Scherer, S. Quake, "Monolithic microfabricated valves and pumps by multilayer soft lithography" Science 288113 (2000)

2. J. Joannopoulos, R. Meade, J. Winn, Photonic Crystals: Molding the Flow of Light. (Princeton University Press, Princeton, New Jersey 1995).

3. M. Loncar, D. Nedeljkovic, T. Doll, J. Vuckovic, A. Scherer, T. Pearsall, "Waveguiding in Planar Photonic Crystals" App Phys Lett. 77 1937 (2000).

4. D. Lide, Ed., Handbook of Chemistry and Physics. (CRC Press, Boca Raton, ed. 79, 1999). 